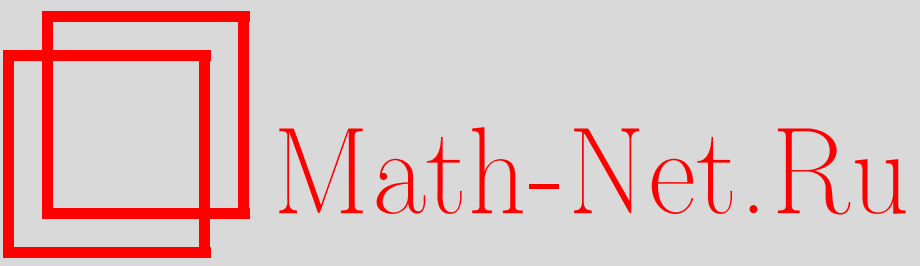

А. А. Белавин, Р. А. Усманов, Минимальная решеточная модель $L M(3,4)$ и двумерная модель Изинга с цилиндрическими граничными условиями, $T M \Phi$, 2001, том 126, номер 1, 63-83

DOI: https://doi.org/10.4213/tmf415

Использование Общероссийского математического портала Math-Net.Ru подразумевает, что вы прочитали и согласны с пользовательским соглашением

http: //www.mathnet.ru/rus/agreement

Параметры загрузки:

IP: 54.198 .67 .100

26 апреля 2023 г., $15: 36: 32$ 
ТЕОРЕТИЧЕСКАЯ

И МАТЕМАТИЧЕСКАЯ

ФИЗИКА

Том 126, № 1

январь, 2001

(C) 2001 г.

А. А. Белавин*, Р. А. Усманов*

\section{МИНИМАЛЬНАЯ РЕШЕТОЧНАЯ МОДЕЛЬ $L M(3,4)$ И ДВУМЕРНАЯ МОДЕЛЬ ИЗИНГА С ЦИЛИНДРИЧЕСКИМИ ГРАНИЧНЫМИ УСЛОВИЯМИ}

Рассматриваются интегрируемая модель $X X Z$ со специальными открытыми граничными условиями и одномерные квантовые цепочки Изинга с четырьмя разными граничными условиями. Показано, что каждая из цепочек Изинга совпадает с получаемой в результате квантово-групповой редукции модели $X X Z$ минимальной решеточной моделью $L M(3,4)$, причем число узлов в последней определяется типом граничных условий. Установлена связь между двумерной моделью Изинга с четырьмя разными типами граничных условий и моделью $\operatorname{LM}(3,4)$.

\section{1. ВВЕДЕНИЕ}

В работах [1], [2] были введены и изучены конечные трансфер-матрицы критической модели Изинга, отвечаюшие четырем разным цилиндрическим граничным условиям. Было показано, что в каждом конкретном случае эти матришы, зависяшие от спектрального параметра, образуют коммутирующее семейство и удовлетворяют одному и тому же функциональному уравнению, которое позволяет найти их собственные значения.

Как будет показано ниже, логарифмическая производная трансфер-матрицы, вычисленная при нулевом значении спектрального параметра, совпадает с гамильтонианом одномерной квантовой цепочки Изинга с некоторыми граничными членами. Последние для каждого типа граничных условий двумерной модели свои. Собственные значения гамильтониана цепочки Изинга в точности совпадают с определенными собственными значениями гамильтониана цепочки $X X Z$ с открытыми граничными условиями при специальном значении параметра анизотропии последней [3]-[5].

В работе [6] введено понятие квантово-групповой редукции в интегрируемой $X X Z$-модели с открытыми граничными условиями при параметре анизотропии, равном корню из единицы. Там же было показано (см. также [7]), что в результате квантово-групповой редукции конфиигурационного пространства $X X Z$-модели получается

\footnotetext{
${ }^{*}$ Институт теоретической физики им. Л. Д. Ландау РАН, Черноголовка, Московская обл., Россия
} 
минимальная модель интегрируемой решеточной теории $L M(p, p+1) \quad(p-$ параметр цепочки). Последняя в термодинамическом пределе есть просто $M(p, p+1)$, минимальная модель конформной теории поля с вирасоровским центральным зарядом $c=1-$ $6 /(p(p+1))$.

Интегрируемость модели $X X Z$ с открытыми граничными условиями проявляется в наличии коммутируюшего семейства трансфер-матриц, найденного Скляниным [8], [9]. Трансфер-матрицы Склянина модели $X X Z$ после квантово-групповой редукции также удовлетворяют некоторому функциональному уравнению [7]. Это уравнение в случае $p=3$ совпадает с функциональным уравнением для трансфер-матриш Изинга, что указывает на эквивалентность моделей Изинга и $L M(3,4)$.

В данной работе мы находим уже упомянутую связь между критической моделью Изинга на двумерной решетке с граничными условиями, введенными в [2], и одномерными квантовыми цепочками Изинга. Кроме того, следуя [10], мы находим условия, при которых имеет место точное совпадение моделей $L M(3,4)$ и этих одномерных цепочек. А именно, справедливы следуюшие утверждения.

1. Цепочка $X X Z$ с нечетным числом узлов $2 L+1$ после квантово-групповой редукции конфигурационного пространства эквивалентна цепочке Изинга с $L$ узлами и смешанными граничными условиями.

2. Конфигурационное пространство цепочки $X X Z$ с четным числом узлов $2 L$ после квантово-групповой редукции можно разложить в прямую сумму двух подпространств одинаковой размерности $2^{L-1}$, образующих два разных представления алгебры Темперли-Либа $T_{2 L-1}$ и являющихся собственными подпространствами оператора Казимира групшы $U_{q}(s l(2))$. Обозначим подпространство, соответствуюшее собственному значению $\left(S^{2}\right)_{q}=\sqrt{2}$, через $V_{1}$, а подпространство, соответствуюшее собственному значению $\left(S^{2}\right)_{q}=0$, через $V_{0}$. Тогда отождествление производится следующим образом:

а) совокупность собственных значений гамильтониана цепочки $X X Z$ с $2 L$ узлами, вычисленных на векторах из $V_{0}$, совпадает со спектром цепочки Изинга с $L-1$ узлами и граничными условиями $(++)$, если $L$ четное, и граничными условиями $(+-)$, если $L$ нечетное;

б) совокупность собственных значений гамильтониана цепочки $X X Z$ с $2 L$ узлами, вычисленных на векторах из $V_{1}$, совпадает со спектром цепочки Изинга с $L-1$ узлами и граничными условиями $(++)$, если $L$ нечетное, и граничными условиями $(+-)$, если $L$ четное.

3. Спектр цепочки Изинга с $L$ узлами и открытыми граничными условиями совпадает с объединенным спектром цепочек Изинга с $L-1$ узлами и граничными условиями $(++)$ и $(+-)$.

Тем самым мы устанавливаем связь между интегрируемой моделью $X X Z$ и моделью Изинга на двумерной решетке с разными граничными условиями.

В разделе 2 определяется модель Изинга с различными цилиндрическими граничными условиями. В разделе 3 вводится семейство трансфер-матриц для четырех типов граничных условий. В разделе 4 найдена связь между введенными трансфер-матрицами и гамильтонианами одномерных цепочек Изинга. Затем в разделе 5 вводится интегри- 
руемая $X X Z$-модель со специальными открытыми граничными условиями. В разделе 6 дается определение алгебры Темперли-Либа и исследуются две ее реализации в терминах динамических переменных цепочек Изинга и $X X Z$. В разделе 7 проводится отождествление цепочки Изинга и модели $L M(3,4)$. В приложениях доказаны некоторые утверждения, использованные в основном тексте.

\section{2. ОСНОВНЫЕ ОПРЕДЕЛЕНИЯ}

Рассмотрим два типа двумерных решеток Изинга [2]:

1) конечная квадратная решетка, повернутая на $45^{\circ}$, причем в каждой строке содержится либо $L$, либо $L-1$ квадратов;

2) такая же решетка, но с $L$ квадратами в каждой строке.

По вертикали каждая решетка содержит по $L^{\prime}$ квадратов в ряду. Мы будем отождествлять первую строку квадратов с $L^{\prime}+1$ строкой (цилиндрические граничные условия). Обозначим решетку первого типа символом $\mathcal{L}$, а решетку второго типа символом $\mathcal{L}^{\prime}$. Обе решетки изображены на рис. 1 . Заметим, что $\mathcal{L}$ состоит из $2 L$, а $\mathcal{L}^{\prime}$ - из $2 L+1$ (зигзагообразных) столбцов. Введем число $N: N=2 L$ для решетки $\mathcal{L}, N=2 L+1$ для решетки $\mathcal{L}^{\prime}$. В дальнейшем будем считать $L$ фиксированным.
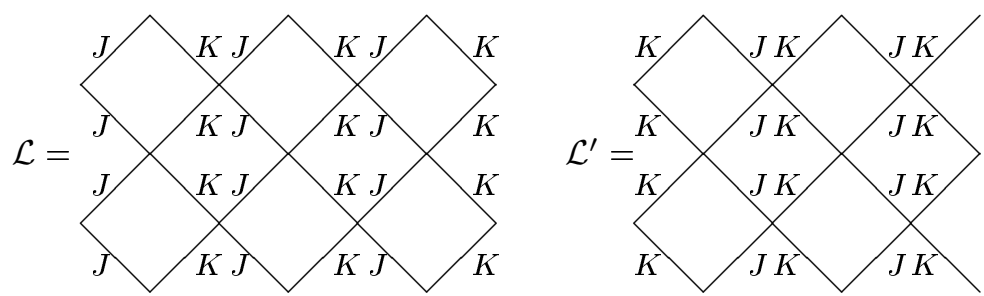

Рис. 1

На этих решетках обычным образом определим модель Изинга, рассматривая каждый узел решетки как спин, принимаюший значения +1 и -1 . Поскольку, в отличие от тороидальной модели, спины, занимаюшие самые крайние узлы в некоторой строке, не равны тождественно, мы можем рассмотреть различные граничные условия:

++ : выбираем решетку $\mathcal{L}$ и полагаем все спины на левой и правой границах равными +1 ;

$+-:$ выбираем решетку $\mathcal{L}$ и полагаем все спины на левой и правой границах равными +1 и -1 , соответственно;

смешанные условия: выбираем решетку $\mathcal{L}^{\prime}$ и полагаем все спины на левой границе равными +1 , причем на значения спинов на правой границе никаких ограничений не налагаем;

свободные условия: выбираем решетку $\mathcal{L}$, причем на значения спинов никаких ограничений не налагаем.

\section{3. ТРАНСФЕР-МАТРИЦЫ}

Обозначая набор спинов в одном ряду, не зафиксированных граничными условиями,

3 Теоретическая и математическая физика, т. 126, № 1, 2001 г. 
через $\Phi$, определим трансфер-матрищы следующим образом:

1) для свободных граничных условий:

$$
T_{\Phi, \Phi^{\prime}}=\sum_{\Phi^{\prime \prime}} \exp \left(J \sum_{j=1}^{L} \sigma_{j}^{\prime \prime}\left(\sigma_{j}+\sigma_{j}^{\prime}\right)+K \sum_{j=1}^{L} \sigma_{j+1}^{\prime \prime}\left(\sigma_{j}+\sigma_{j}^{\prime}\right)\right)
$$

2) для фиксированных граничных условий:

$$
T_{\Phi, \Phi^{\prime}}=\sum_{\Phi^{\prime \prime}} \exp \left(2 J \sigma_{1}^{\prime \prime}+K \sum_{j=1}^{L-1} \sigma_{j}^{\prime \prime}\left(\sigma_{j}+\sigma_{j}^{\prime}\right)+J \sum_{j=1}^{L-1} \sigma_{j+1}^{\prime \prime}\left(\sigma_{j}+\sigma_{j}^{\prime}\right)+2 \tau K \sigma_{L}^{\prime \prime}\right)
$$

где $\tau= \pm 1$ соответственно для граничных условий $(++)$ и $(+-)$;

3) для смешанных граничных условий:

$$
T_{\Phi, \Phi^{\prime}}=\sum_{\Phi^{\prime \prime}} \exp \left(2 K \sigma_{1}^{\prime \prime}+J \sum_{j=1}^{L} \sigma_{j}^{\prime \prime}\left(\sigma_{j}+\sigma_{j}^{\prime}\right)+K \sum_{j=1}^{L} \sigma_{j+1}^{\prime \prime}\left(\sigma_{j}+\sigma_{j}^{\prime}\right)\right) .
$$

Здесь везде $\Phi, \Phi^{\prime \prime}$ и $\Phi^{\prime}$ - три последовательных ряда спинов, по $\Phi^{\prime \prime}$ производится суммирование. В случае свободных и фиксированных граничных условий трансфер-матрицы определяются на решетке $\mathcal{L}$, в случае смешанных - на решетке $\mathcal{L}^{\prime}$.

Трансфер-матрицы, определенные выше, обладают свойством коммутативности, т.е.

$$
T(J, K) T\left(J^{\prime}, K^{\prime}\right)=T\left(J^{\prime}, K^{\prime}\right) T(J, K)
$$

если

$$
\operatorname{sh}(2 K) \operatorname{sh}(2 J)=\operatorname{sh}\left(2 K^{\prime}\right) \operatorname{sh}\left(2 J^{\prime}\right) .
$$

Доказательство этого факта приведено в приложении А.

Мы будем рассматривать критическую модель Изинга, ипоэтому $\operatorname{sh}(2 J) \operatorname{sh}(2 K)=1$. Следуя Бакстеру, будем использовать параметризацию

$$
\operatorname{sh}(2 J)=\operatorname{ctg} u, \quad \operatorname{sh}(2 K)=\operatorname{tg} u,
$$

где $u$ - спектральный параметр, $0<u<\pi / 2$.

Как показано в [2], матрица $T(u)$ удовлетворяет функциональному уравнению

$$
T(2 u) T\left(2 u+\frac{\pi}{2}\right)=\frac{\cos ^{2(N+1)}(2 u)-\sin ^{2(N+1)}(2 u)}{\cos (4 u)} \frac{2^{N}(-1)^{L}}{(\sin (2 u) \cos (2 u))^{N}}
$$

в случае фиксированных граничных условий и уравнению

$$
T(2 u) T\left(2 u+\frac{\pi}{2}\right)=\frac{\cos ^{2(N+1)}(2 u)-\sin ^{2(N+1)}(2 u)}{\cos (4 u)} \frac{2^{N+2}(-1)^{L}}{(\sin (2 u) \cos (2 u))^{N}}
$$

в случае свободных и смешанных граничных условий. 
МИНИМАЛЬНАЯ РЕШЕТОЧНАЯ МОДЕЛЬ $L M(3,4)$ И ДВУМЕРНАЯ МОДЕЛЬ ИЗИНГА 67

\section{4. СВЯЗЬ ТРАНСФЕР-МАТРИЦ С ГАМИЛЬТОНИАНОМ ОДНОМЕРНОЙ ЦЕПОЧКИ ИЗИНГА}

Рассмотрим гамильтониан одномерной квантовой цепочки Изинга с $L$ узлами

$$
H_{\text {Ising }}^{0}(L)=\sum_{j=1}^{L-1} \sigma_{j}^{z} \sigma_{j+1}^{z}+\sum_{j=1}^{L} \sigma_{j}^{x} .
$$

Этот гамильтониан связан с трансфер-матрицей $T(u)$ модели Изинга со свободными граничными условиями следующим образом:

$$
H_{\text {Ising }}^{0}(L)=T^{-1}(0) \dot{T}(0)-\text { "сингулярные члены”. }
$$

Здесь $T^{-1}(u)$ - обратная матрица, а $\dot{T}(u)$ - производная от матрицы $T(u)$ по параметру $u$.

Для других граничных условий логарифмическая производная в нуле слегка модифицируется. В данной работе мы показываем, что

1) в случае граничных условий $(++)$ получается гамильтониан цепочки Изинга с $L-1$ узлами

$$
H_{\mathrm{I} \text { sing }}^{++}(L)=H_{\mathrm{I} \operatorname{sing}}^{0}(L-1)+\sigma_{1}^{z}+\sigma_{L-1}^{z},
$$

2) в случае граничных условий $(+-)$ получается гамильтониан цепочки Изинга с $L-1$ узлами

$$
H_{\text {Ising }}^{+-}(L)=H_{\text {Ising }}^{0}(L-1)+\sigma_{1}^{z}-\sigma_{L-1}^{z}
$$

3) в случае смешанных граничных условий получается гамильтониан цепочки Изинга с $L$ узлами

$$
H_{\mathrm{Ising}}^{\text {cмeш }}(L)=H_{\mathrm{Ising}}^{0}(L)+\sigma_{1}^{z} .
$$

Здесь $H_{\text {Ising }}^{0}$ - гамильтониан модели со свободными граничными условиями.

Доказательство перечисленных фактов приведено в приложении Б.

\section{5. МИНИМАЛЬНЫЕ МОДЕЛИ ИНТЕГРИРУЕМЫХ РЕШЕТОЧНЫХ ТЕОРИЙ}

Рассмотрим одномерную $X X Z$-цепочку со свободными граничными условиями [3]

$$
\begin{gathered}
H_{X X Z}=\sum_{n=1}^{N-1}\left[\sigma_{n}^{+} \sigma_{n+1}^{-}+\sigma_{n}^{-} \sigma_{n+1}^{+}+\frac{q+q^{-1}}{4} \sigma_{n}^{z} \sigma_{n+1}^{z}+\frac{q-q^{-1}}{4}\left(\sigma_{n}^{z}-\sigma_{n+1}^{z}\right)\right], \\
\sigma_{n}^{ \pm}=1 \otimes \cdots \otimes \sigma^{ \pm} \otimes \cdots \otimes 1, \\
\sigma_{n}^{z}=1 \otimes \cdots \otimes \sigma^{z} \otimes \cdots \otimes 1 \\
\sigma^{+}=\left(\begin{array}{ll}
0 & 1 \\
0 & 0
\end{array}\right), \quad \sigma^{-}=\left(\begin{array}{ll}
0 & 0 \\
1 & 0
\end{array}\right), \quad \sigma^{z}=\left(\begin{array}{cc}
1 & 0 \\
0 & -1
\end{array}\right) .
\end{gathered}
$$


Как показано в [6], гамильтониан $H_{X} X Z$ коммутирует с квантовой группой $U_{q}(s l(2))$, в том числе и с ее генераторами $X, Y, H$, которые определяются формулами

$$
\begin{aligned}
& X=\sum_{n=1}^{N} q^{\frac{1}{2}\left(\sigma_{1}^{z}+\cdots+\sigma_{n-1}^{z}\right)} \sigma_{n}^{+} q^{-\frac{1}{2}\left(\sigma_{n+1}^{z}+\cdots+\sigma_{N}^{z}\right)}, \\
& Y=\sum_{n=1}^{N} q^{\frac{1}{2}\left(\sigma_{1}^{z}+\cdots+\sigma_{n-1}^{z}\right)} \sigma_{n}^{-} q^{-\frac{1}{2}\left(\sigma_{n+1}^{z}+\cdots+\sigma_{N}^{z}\right)}, \\
& H=\sum_{n=1}^{N} \frac{\sigma_{n}^{z}}{2}
\end{aligned}
$$

и удовлетворяют следуюшим соотношениям коммутации:

$$
[H, X]=X, \quad[H, Y]=-Y, \quad[X, Y]=\frac{q^{2 H}-q^{-2 H}}{q-q^{-1}} .
$$

Кроме того, с квантовой группой $U_{q}(s l(2))$ коммутируют также плотности

$$
H_{n}=\sigma_{n}^{+} \sigma_{n+1}^{-}+\sigma_{n}^{-} \sigma_{n+1}^{+}+\frac{q+q^{-1}}{4} \sigma_{n}^{z} \sigma_{n+1}^{z}+\frac{q-q^{-1}}{4}\left(\sigma_{n}^{z}-\sigma_{n+1}^{z}\right),
$$

где $n=1, \ldots, N-1$.

Благодаря указанной симметрии гамильтониана его спектр можно классифицировать согласно теории представлений алгебры $U_{q}(s l(2))$.

Теория представлений алгебры $U_{q}(s l(2))$ хорошо изучена для чисел $q$, не равных корню из единицы. В данном случае представления этой алгебры эквивалентны представлениям обычной алгебры $U(s l(2))$, т.е. конфигурационное пространство $\left(\mathbb{C}^{2}\right)^{N}$ цепочки спинов разлагается в прямую сумму неприводимых представлений $\rho_{j}(j-$ старший вес $)$, каждому из которых однозначно соответствует некоторое представление $s l(2)$. Например, если $N=4$, то $\left(\mathbb{C}^{2}\right)^{4}$ можно разложить в сумму $\rho_{2}+3 \rho_{1}+2 \rho_{0}$.

Мы же будем изучать случай $q^{p+1}=-1[6],[11]$. В этом случае генераторы $X$ и $Y$ нильпотентны:

$$
X^{p+1}=0, \quad Y^{p+1}=0,
$$

и возникает совсем другая картина представлений. Например, если $q^{4}=-1$ и $N=4$, то $X^{4}=0$ и $Y^{4}=0$. Пространство $\left(\mathbb{C}^{2}\right)^{4}$ разлагается в сумму одного "плохого" 8-мерного представления $\left(\rho_{2}, \rho_{1}\right)$ типа I и четырех "хороших" представлений $2 \rho_{1}+2 \rho_{0}$ типа II [6]. Имеется изоморфизм представлений типа II и обычных представлений алгебры $U(s l(2))$. Представление же типа I можно рассматривать как результат склеивания двух представлений $\rho_{2}$ и $\rho_{1}$. Оно приводимо (но не является вполне приводимым), поскольку содержит 3 -мерное инвариантное подпространство.

В обшем случае $q^{p+1}=-1[6],[11]$ конфигурационное пространство разлагается в сумму "плохих" представлений типа I со старшими весами $S_{z} \geqslant p / 2$ и "хороших" представлений типа II со старшими весами $S_{z}<p / 2$, не являюшихся подпространствами 
каких-либо "плохих". Старшие векторы $v_{j}$ "хороших" представлений можно охарактеризовать условием

$$
v_{j} \in V_{p} \equiv \operatorname{Ker} X / \operatorname{Im} X^{p}
$$

Поскольку алгебра $U_{q}(s l(2))$ коммутирует с гамильтонианом $H_{X X Z}$, мы можем естественным образом ограничить действие последнего на пространство $V_{p}$. Полученная в результате этой квантово-групповой редукции модель называется моделью $L M(p, p+1)$ [7].

Число представлений веса $j<p / 2$ в разложении $\left(\mathbb{C}^{2}\right)^{N}$ равно числу ограниченных путей длины $N$ с началом в нуле и концом в $j$. Ограниченность означает, что путь не должен пересекать прямые $j=0$ и $j=p / 2$. Например, если $p=3$ и $N$ нечетно, то допустимы только пути с концом в $j=1 / 2$, и поэтому число путей равно $2^{N-1}$.

С гамильтонианом цепочки $X X Z$ связана трансфер-матрица Склянина [8], [9]

$$
T_{1 / 2}(u)=(-1)^{N} \operatorname{tr}\left(e^{-\sigma^{z}(u+\eta)} L(u) e^{\sigma^{z} u} L^{t \otimes t}(u)\right),
$$

где $L(u)$ - матрица монодромии:

$$
L(u)=R_{N}(u) \ldots R_{1}(u) .
$$

Матрица $R(u)$ в свою очередь дается выражением

$$
\left(\begin{array}{cc}
\left(\begin{array}{cc}
\sin (u+\eta) & 0 \\
0 & \sin u
\end{array}\right) & \left(\begin{array}{cc}
0 & 0 \\
\sin \eta & 0
\end{array}\right) \\
\left(\begin{array}{cc}
0 & \sin \eta \\
0 & 0
\end{array}\right) & \left(\begin{array}{cc}
\sin u & 0 \\
0 & \sin (u+\eta)
\end{array}\right)
\end{array}\right)
$$

Имеет место соотношение

$$
H_{X X Z}=\left.\frac{\sin \eta}{2} \frac{d \ln T_{1 / 2}(u)}{d u}\right|_{u=0}+\frac{\sin ^{2} \eta}{2 \cos \eta}-\frac{N}{2} \cos \eta .
$$

Трансфер-матрицы коммутируют между собой при разных значениях спектрального параметра, а следовательно, коммутируют и с гамильтонианом, т.е.

$$
\left[T_{1 / 2}(u), T_{1 / 2}(v)\right]=0, \quad\left[T_{1 / 2}(u), H_{X X Z}\right]=0
$$

Как показано в [7], после квантово-групповой редукции матрица $T_{1 / 2}(u)$ удовлетворяет следующему функциональному уравнению:

$$
T_{1 / 2}(u) T_{1 / 2}\left(u+\frac{\pi}{4}\right)=2^{-2 N+1} \frac{\cos ^{2(N+1)}(2 u)-\sin ^{2(N+1)}(2 u)}{\cos (4 u)} .
$$

Сравнивая уравнения (2) и (9), мы видим, что в случае четных $N$ и фиксированных граничных условий матрицы

$$
T_{1 / 2}(u) \quad \text { и } 2^{\frac{1}{2}} \frac{1}{2^{2 N}}(\sin (4 u))^{L} T_{\text {Ising }}(2 u)
$$


удовлетворяют одному и тому же функциональному уравнению. Здесь трансфер-матрица модели Изинга с фиксированными граничными условиями обозначена через $T_{\text {Ising }}(u)$, а не $T(u)$, как это было в (2). Отсюда следует, что их собственные значения одинаковы, и поэтому обе матришы эквивалентны друг другу.

В случае четных $N$ и свободных граничных условий одному и тому же функциональному уравнению удовлетворяют матрицы

$$
T_{1 / 2}(u) \text { и } 2^{\frac{1}{2}} \frac{1}{2^{2 N+1}}(\sin (4 u))^{L} T_{\text {Ising }}(2 u),
$$

где матрица $T_{\mathrm{Ising}}(u)$ - трансфер-матрица модели Изинга со свободными граничными условиями.

Аналогично в случае нечетных $N$, т.е. для смешанных граничных условий, матрицы

$$
T_{1 / 2}(u) \text { и } 2^{\frac{1}{2}} \frac{1}{2^{N}}(\sin (2 u))^{L+1}(\cos (2 u))^{L} T_{\text {Ising }}(2 u)
$$

также удовлетворяют одному и тому же функциональному уравнению, где $T_{\mathrm{Ising}}(u)-$ трансфер-матрица модели Изинга со смешанными граничными условиями.

Еще раз подчеркнем, что цепочка $X X Z$ содержит $N$ узлов, $N=2 L$ для свободных и для фиксированных граничных условий и $N=2 L+1$ для смешанных граничных условий.

То, что уравнения совпадают, свидетельствует в пользу идентичности моделей (множители перед матрищей $T_{\text {Ising }}(2 u)$ несушественны, поскольку они влияют только на ее нормировку). Ниже мы выясним, как именно происходит отождествление.

\section{6. АЛГЕБРА ТЕМПЕРЛИ-ЛИБА}

Напомним определение алгебры Темперли-Либа. Она представляет из себя алгебру с образующими $e_{i}, i=1, \ldots, n$, удовлетворяюшими следуюшим соотношениям:

$$
e_{i} e_{i \pm 1} e_{i}=e_{i}, \quad e_{i}^{2}=\sqrt{\left(q+q^{-1}\right)} e_{i}, \quad\left[e_{i}, e_{j}\right]=0, \quad|i-j|>1 .
$$

Алгебру Темперли-Либа с $n$ образуюшими будем обозначать символом $T_{n}$. Везде в дальнейшем будем считать, что $q=e^{i \pi / 4}$.

Существуют реализации алгебры Темперли-Либа в терминах динамических переменных моделей Изинга и $X X Z$. А именно, можно проверить, что

1) выражения

$$
\begin{aligned}
e_{2 i-1} & =\frac{1}{\sqrt{2}}\left(\sigma_{i}^{z} \sigma_{i+1}^{z}+1\right), & i & =1, \ldots, L, \\
e_{2 i} & =\frac{1}{\sqrt{2}}\left(\sigma_{i}^{x}+1\right), & i & =1, \ldots, L-1,
\end{aligned}
$$

дают реализацию алгебры $T_{2 L-1}$ (удовлетворяют соотношениям (10)); 
МИНИМАЛЬНАЯ РЕШЕТОЧНАЯ МОДЕЛЬ $L M(3,4)$ И ДВУМЕРНАЯ МОДЕЛЬ ИЗИНГА 71

2) выражения

$$
\begin{array}{rlrl}
e_{1} & =\frac{1}{\sqrt{2}}\left(\sigma_{1}^{z}+1\right), & \\
e_{2 i} & =\frac{1}{\sqrt{2}}\left(\sigma_{i}^{z} \sigma_{i+1}^{z}+1\right), \quad i=1, \ldots, L, \\
e_{2 i-1} & =\frac{1}{\sqrt{2}}\left(\sigma_{i}^{x}+1\right), \quad i=2, \ldots, L
\end{array}
$$

дают реализацию алгебры $T_{2 L}$;

3) выражения

$$
\begin{array}{rlrl}
e_{1} & =\frac{1}{\sqrt{2}}\left(\sigma_{1}^{z}+1\right), & \\
e_{2 i-1} & =\frac{1}{\sqrt{2}}\left(\sigma_{i}^{z} \sigma_{i+1}^{z}+1\right), \quad i=2, \ldots, L, \\
e_{2 i} & =\frac{1}{\sqrt{2}}\left(\sigma_{i}^{x}+1\right), \quad i=1, \ldots, L-1, \\
e_{2 L-1} & =\frac{1}{\sqrt{2}}\left(\sigma_{L-1}^{z}+1\right)
\end{array}
$$

дают реализацию алгебры $T_{2 L-1}$;

4) выражения

$$
\begin{aligned}
e_{1} & =\frac{1}{\sqrt{2}}\left(\sigma_{1}^{z}+1\right), \\
e_{2 i-1} & =\frac{1}{\sqrt{2}}\left(\sigma_{i}^{z} \sigma_{i+1}^{z}+1\right), \quad i=2, \ldots, L, \\
e_{2 i} & =\frac{1}{\sqrt{2}}\left(\sigma_{i}^{x}+1\right), \quad i=1, \ldots, L-1, \\
e_{2 L-1} & =\frac{1}{\sqrt{2}}\left(-\sigma_{L-1}^{z}+1\right)
\end{aligned}
$$

дают реализацию алгебры $T_{2 L-1}$.

Используя эти выражения, приводим гамильтониан модели Изинга для всех четырех граничных условий к виду

$$
H_{\text {Ising }}=\sum_{i=1}^{N-1}\left(e_{i} \sqrt{2}-1\right)
$$

где $N=2 L$ для свободных и фиксированных граничных условий и $N=2 L+1$ для смешанных граничных условий.

В терминах динамических переменных модели $X X Z$ выражения

$$
e_{i}=-H_{i}+\frac{\sqrt{2}}{4}
$$


где $i=1, \ldots, N-1$, дают реализацию алгебры $T_{N-1}$, а гамильтониан цепочки $X X Z \mathrm{c}$ $N$ узлами принимает вид

$$
H_{X X Z}=-\sum_{i=1}^{N-1}\left(e_{i}-\frac{\sqrt{2}}{4}\right) .
$$

Таким образом, гамильтонианы моделей $X X Z$ и Изинга в терминах образующих алгебры Темперли-Либа имеют одинаковый вид. Если обе реализации осуществляют одно и то же представление этой алгебры, то они эквивалентны. В следующем разделе мы найдем условия, при которых эта эквивалентность имеет место.

\section{7. ОТОЖДЕСТВЛЕНИЕ МОДЕЛИ $L M(3,4)$ И МОДЕЛИ ИЗИНГА}

7.1. Цепочка $X X Z$ с нечетным числом узлов и цепочка Изинга со смешанными граничными условиями. Рассмотрим цепочку $X X Z$ с $N=2 L+1$ узлами и цепочку Изинга с $L$ узлами и смешанными граничными условиями. Обеим цепочкам соответствует одна и та же алгебра $T_{2 L}$. Плотности $H_{i}$ гамильтониана модели $X X Z$ коммутируют с квантовой группой $U_{q}(s l(2))$. Отсюда следует, что конфигурационное пространство модели $L M(3,4)$ образует представление алгебры $T_{2 L}$. Это представление имеет размерность $2^{L}$ и является неприводимым.

С другой стороны, представление алгебры Темперли-Либа, реализуемое моделью Изинга, имеет ту же размерность и также является неприводимым, поскольку действием операторов $\sigma_{x}$ из заданного вектора можно получить все конфигурационное пространство модели Изинга.

Учитывая тот факт, что $T_{2 L}$ с $q=e^{i \pi / 4}$ имеет только одно неприводимое представление размерности $2^{L}[12]$, а также то, что гамильтонианы обеих моделей имеют одинаковый вид в терминах образуюших $T_{2 L}$, делаем вывод, что обе модели эквивалентны. Проверить это можно численно, сравнивая собственные значения оператора

$$
\sum_{i=1}^{N-1} e_{i}
$$

вычисленные на векторах конфигурационного пространства $L M(3,4)$ и на векторах из конфигурационного пространства Изинга для небольших $L$.

7.2. Цепочка $X X Z$ с четным числом узлов и цепочки Изинга с фиксированными граничными условиями. Теперь рассмотрим цепочку $X X Z \mathrm{c} N=2 L$ узлами и цепочку Изинга с $L-1$ узлами и фиксированными граничными условиями. В предыдущем разделе было показано, что обеим цепочкам соответствует одна и та же алгебра Темперли-Либа $T_{N-1}$.

В этом случае размерность конфигурационного пространства каждого из гамильтонианов Изинга вдвое меньше размерности конфигурационного пространства $V_{3}$ модели $L M(3,4)$. Пространство $V_{3}$ имеет размерность $2^{L}$ и разбивается на два подпространства, каждое из которых является собственным подпространством оператора Казимира

$$
\left(S^{2}\right)_{q}=Y X+\left(\frac{q^{H+\frac{1}{2}}-q^{-H-\frac{1}{2}}}{q-q^{-1}}\right)^{2}-\left(\frac{q^{\frac{1}{2}}-q^{-\frac{1}{2}}}{q-q^{-1}}\right)^{2}
$$


одно из них отвечает собственному значению $\left(S^{2}\right)_{q}=0$, другое - собственному значению $\left(S^{2}\right)_{q}=\sqrt{2}$. Будем обозначать первое подпространство символом $V_{0}$, а второе символом $V_{1}$. Поскольку оператор Казимира коммутирует с алгеброй $T_{2 L-1}$, подпространства $V_{0}$ и $V_{1}$ образуют представления последней. Оба представления неприводимы, причем размерность каждого из них равна $2^{L-1}$, что совпадает с размерностью конфигурационного пространства каждой из двух цепочек Изинга.

Как показано в [10],

$$
2^{2 N} q^{-N} e^{-2 i N u} T_{1 / 2}(u \rightarrow-i \infty)=\left\{\left(q-q^{-1}\right)^{2}\left(S^{2}\right)_{q}+\left(q+q^{-1}\right)\right\},
$$

откуда видно, что пространства $V_{0}$ и $V_{1}$ являются собственными подпространствами матрицы, стояшей в левой части последнего равенства, отвечаюшими двум ее собственным значениям. Эти собственные значения равны \pm 1 .

Алгебра $T_{2 L-1}$ с $q=e^{i \pi / 4}$ обладает только двумя неприводимыми представлениями размерности $2^{L-1}[12]$. Поэтому конфигурационное пространство цепочки Изинга с граничными условиями $(++)$ должно быть изоморфно одному из подпространств $V_{0}$ или $V_{1}$. То же самое можно сказать и про конфигурационное пространство цепочки Изинга с граничными условиями $(+-)$.

В работе [2] было показано, что $T_{\text {Ising }}(-i \infty)= \pm 2^{L}$, где верхний знак отвечает граничным условиям $(++)$, а нижний - граничным условиям $(+-)$. После квантово-групповой редукции матрицы $2^{1-2 N}(\sin (4 u))^{L} T_{\text {Ising }}(2 u)$ и $T_{1 / 2}(u)$ становятся эквивалентными. Поэтому, переходя к пределу $u \rightarrow-i \infty$ в равенстве

$$
T_{1 / 2}(u)=2^{1-2 N}(\sin (4 u))^{L} T_{\text {Ising }}(2 u)
$$

и подставляя $q=e^{i \pi / 4}$, получим

$$
\frac{1}{2^{L}} T_{\text {Ising }}(-i \infty)=(-1)^{L}\left\{-\sqrt{2}\left(S^{2}\right)_{q}+1\right\} .
$$

Поскольку собственные значения оператора Казимира есть 0 и $\sqrt{2}$, мы приходим к заключению, что, например, для четных $L$ пространству $V_{0}$ соответствует конфигурационное пространство цепочки Изинга с граничными условиями $(++)$, а пространству $V_{1}$ - конфигурационное пространство цепочки Изинга с граничными условиями $(+-)$. Для нечетных $L$ пространству $V_{0}$ соответствуют граничные условия $(+-)$, а пространству $V_{1}$ - граничные условия $(++)$.

7.3. Цепочка $X X Z$ с четным числом узлов и цепочка Изинга со свободными граничными условиями. Пусть теперь имеются цепочка $X X Z$ с четным числом узлов $N=2 L$ и цепочка Изинга с $L$ узлами и свободными граничными условиями. Им соответствует алгебра $T_{2 L-1}$. Размерности конфигурационных пространств цепочки Изинга и модели $L M(3,4)$ совпадают и равны $2^{L}$.

Введем оператор $C$. В случае свободных граничных условий

$$
C=\sigma_{1}^{x} \sigma_{2}^{x} \ldots \sigma_{L}^{x}
$$


Очевидно, что $C^{2}=1$. Поэтому собственные значения оператора $C$ есть \pm 1 . Этот оператор коммутирует с гамильтонианом $H_{\text {Ising. }}^{0}$ Следовательно, конфигурационное пространство цепочки Изинга со свободными граничными условиями разбивается на два сектора, соответствуюших двум собственным значениям оператора $C$. Будем обозначать эти два сектора $C_{+}$и $C_{-}$.

В работе [10] показано, что оператор $C$ связан с асимптотикой трансфер-матрицы модели Изинга с $L$ узлами и свободными граничными условиями соотношением

$$
C=\frac{1}{2^{L+1}} T_{\text {Ising }}(-i \infty)
$$

Точно так же, как и для случая фиксированных граничных условий, можно получить равенство

$$
C=\frac{1}{2^{L+1}} T_{\text {Ising }}(-i \infty)=(-1)^{L}\left\{-\sqrt{2}\left(S^{2}\right)_{q}+1\right\},
$$

из которого видно, что имеется взаимно однозначное соответствие между секторами в конфигурационном пространстве цепочки Изинга и подпространствами $V_{0}$ и $V_{1}$. А именно, при четных $L$ пространству $V_{0}$ соответствует сектор $C_{+}$, а пространству $V_{1}$ - сектор $C_{-}$. При нечетных $L$ наоборот: пространству $V_{0}$ соответствует сектор $C_{-}$, пространству $V_{1}-$ сектор $C_{+}$.

Таким образом, все утверждения, сформулированные во введении, доказаны.

ПРИЛОЖЕНИЕ А

\section{Коммутативность трансфер-матриц Изинга}

Рассмотрим случай граничных условий $(++)$. Доказательство будем проводить, используя соотношение звезда-треугольник. Для простоты рассмотрим случай одного свободного спина. Произведение трансфер-матриц $T(J, K) T\left(J^{\prime}, K^{\prime}\right)$ можно изобразить следуюшей исходной диаграммой (рис. 2):

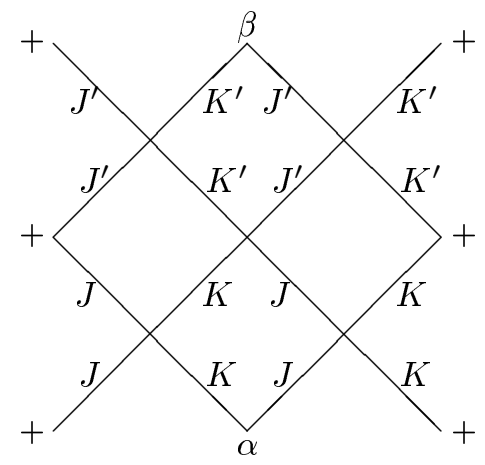

Рис. 2

Здесь $\alpha$ и $\beta$ фиксированы, а по всем остальным неграничным спинам производится суммирование. Мы должны доказать, что эта диаграмма равна диаграмме, изображенной на рис. 3 , получаюшейся из исходной заменой $K \leftrightarrow K^{\prime}, J \leftrightarrow J^{\prime}$. 


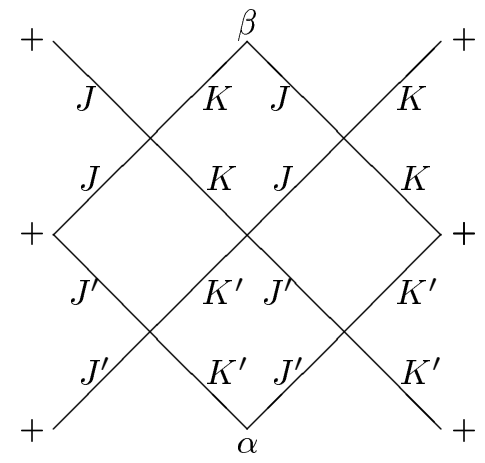

Рис. 3

Для дальнейшего нам понадобятся два тождества, справедливые при $\operatorname{sh}(2 J) \operatorname{sh}(2 K)=$ $\operatorname{sh}\left(2 J^{\prime}\right) \operatorname{sh}\left(2 K^{\prime}\right)$. Одно из них можно изобразить диаграммным равенством

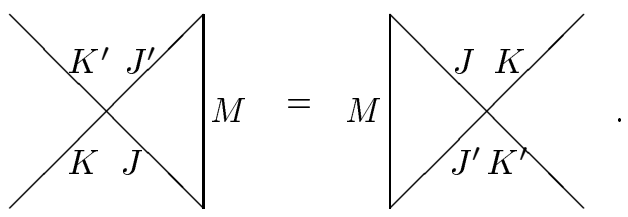

Для доказательства применим соотношение звезда-треугольник к треугольникам $\left(J, J^{\prime}, M\right)$ в обеих частях равенства. Получим (при условии, что $\operatorname{sh}(2 K) \operatorname{sh}(2 J)=$ $\left.\operatorname{sh}\left(2 K^{\prime}\right) \operatorname{sh}\left(2 J^{\prime}\right)\right)$

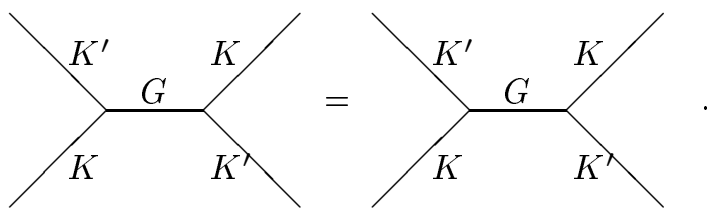

Мы видим, что тождество действительно имеет место.

Второе тождество также представляется в виде равенства двух диаграмм

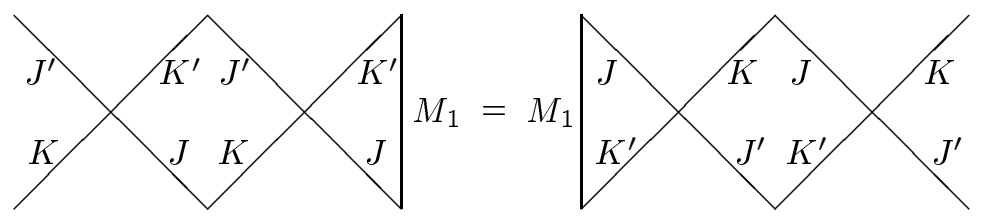

и доказывается аналогично. 
Перейдем теперь непосредственно к доказательству нашего утверждения о коммутативности семейства трансфер-матриц. Используя первое из доказанных тождеств, приведем исходную диаграмму к виду, представленному на рис. 4.

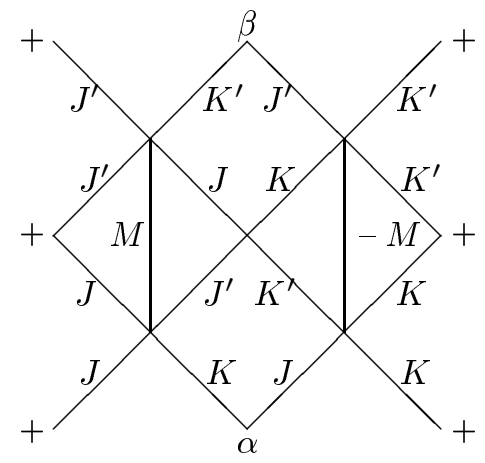

Рис. 4

Естественно, что это можно сделать только при условии $\operatorname{sh}(2 J) \operatorname{sh}(2 K)=\operatorname{sh}\left(2 J^{\prime}\right) \times$ $\operatorname{sh}\left(2 K^{\prime}\right)=\lambda$. Далее применим соотношение звезда-треугольник к двум крайним треугольникам $\left(J, J^{\prime}, M\right)$ и $\left(K, K^{\prime},-M\right)$. В результате получим диаграмму на рис. 5 .

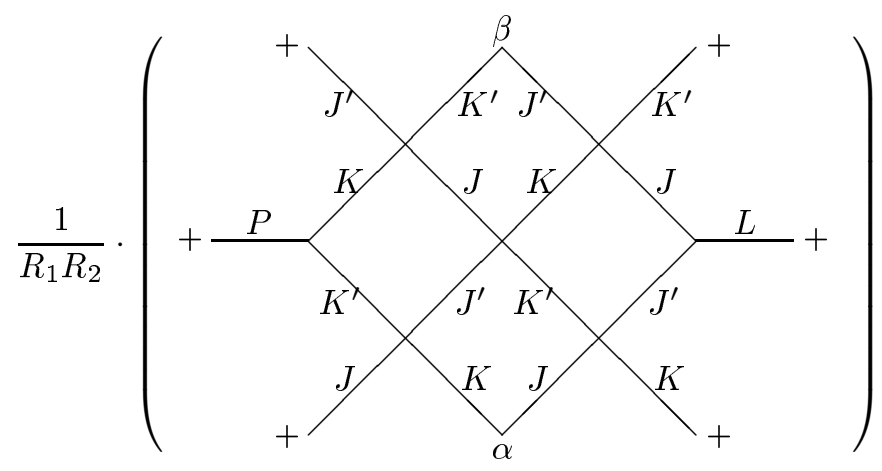

Рис. 5

Здесь $R_{1}, R_{2}$ - множители, возникшие после применения соотношения звезда-треугольник,

$$
\begin{aligned}
& R_{1}^{2}=\frac{2 \lambda^{2}}{\operatorname{sh}(2 J) \operatorname{sh}\left(2 J^{\prime}\right) \operatorname{sh}(2 M)} \\
& R_{2}^{2}=-\frac{2 \lambda^{2}}{\operatorname{sh}(2 K) \operatorname{sh}\left(2 K^{\prime}\right) \operatorname{sh}(2 M)}
\end{aligned}
$$


МИНИМАЛЬНАЯ РЕШЕТОЧНАЯ МОДЕЛЬ $L M(3,4)$ И ДВУМЕРНАЯ МОДЕЛЬ ИЗИНГА 77 а константы связи $P$ и $L$ связаны с $M$ соотношением

$$
\operatorname{sh}(2 P) \operatorname{sh}(2 M)=-\operatorname{sh}(2 L) \operatorname{sh}(2 M)=\lambda .
$$

Теперь применим второе из доказанных нами тождеств к диаграмме на рис. 5. Получим диаграмму, изображенную на рис. 6.

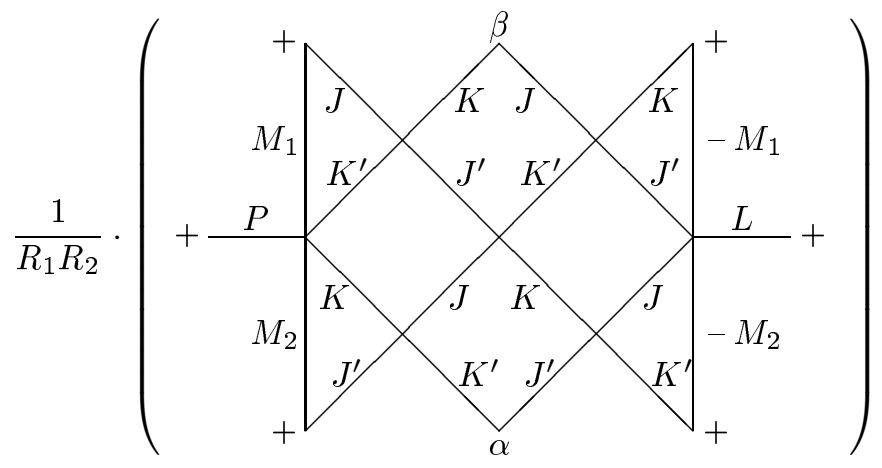

Рис. 6

Очевидно, что $M_{1}$ и $M_{2}$ связаны соотношением $M_{1}=-M_{2}$. Поэтому при вычислении статсуммы они взаимно сокращаются.

Опять применим первое тождество. Получим диаграмму на рис. 7.

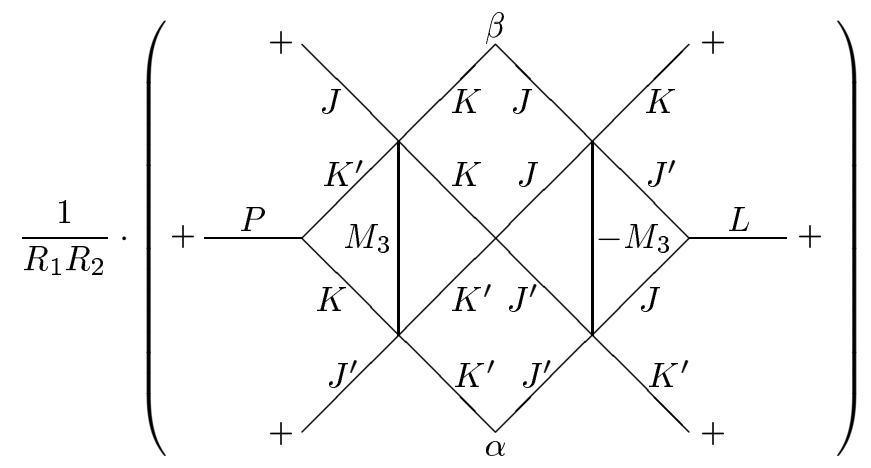

Рис. 7

Легко показать, что константа связи $M_{3}$, введенная здесь, есть $-M$.

Снова применяя соотношение звезда-треугольник, получим диаграмму на рис. 8 . 


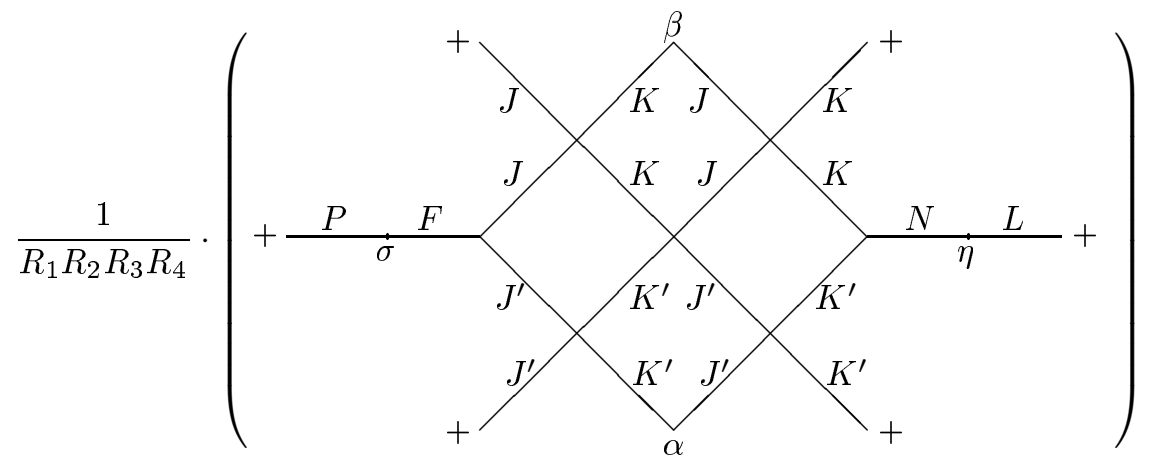

Рис. 8

Здесь

$$
\begin{aligned}
& R_{3}^{2}=\frac{2 \lambda^{2}}{\operatorname{sh}(2 J) \operatorname{sh}\left(2 J^{\prime}\right) \operatorname{sh}(2 M)}, \\
& R_{4}^{2}=-\frac{2 \lambda^{2}}{\operatorname{sh}(2 K) \operatorname{sh}\left(2 K^{\prime}\right) \operatorname{sh}(2 M)},
\end{aligned}
$$

константы связи $F, N$ и $M$ удовлетворяют соотношению

$$
\operatorname{sh}(2 M) \operatorname{sh}(2 N)=-\operatorname{sh}(2 M) \operatorname{sh}(2 F)=\lambda
$$

а по спинам $\eta$ и $\sigma$ производится суммирование.

Видно, что диаграмма на рис. 8 очень похожа на диаграмму на рис. 3 (за исключением обшего множителя и боковых отростков). Покажем, что можно подобрать константы связи $P, F, N$ и $L$, удовлетворяющие соотношениям (А.2) и (А.4), такие, что боковые отростки и общий множитель взаимно сокращаются.

Боковой отросток имеет вид

$$
\mu \frac{N L L}{\eta}+= \begin{cases}2 \operatorname{ch}(L+N), & \mu=+ \\ 2 \operatorname{ch}(L-N), & \mu=-\end{cases}
$$

Положим

$$
N=L+\frac{\pi i}{2}
$$

Тогда

$$
\mu \frac{N \quad L}{\eta}+=\left\{\begin{array}{cl}
2 i \operatorname{sh}(2 L), & \mu=+ \\
0, & \mu=-
\end{array}\right\}=2 i \operatorname{sh}(2 L) \cdot \delta_{\mu,+}
$$


Аналогично, положив

$$
F=P+\frac{\pi i}{2}
$$

получим равенство

$$
+\frac{P \quad F}{\sigma} \nu=\left\{\begin{array}{cc}
2 i \operatorname{sh}(2 P), & \nu=+ \\
0, & \nu=-
\end{array}\right\}=2 i \operatorname{sh}(2 P) \cdot \delta_{\nu,+} .
$$

Поскольку

$$
\operatorname{sh}(2 P)=-\operatorname{sh}(2 L)=\frac{\lambda}{\operatorname{sh}(2 M)}
$$

то диаграмма на рис. 8 сводится к диаграмме на рис. 3 , помноженной на коэффицциент

$$
\frac{1}{R_{1} R_{2} R_{3} R_{4}} \frac{4 \lambda^{2}}{\operatorname{sh}^{2}(2 M)}
$$

Подставляя выражения для $R_{1}, R_{2}, R_{3}$ и $R_{4}$, легко найти, что этот коэффипиент равен единице. Таким образом, коммутативность двух матриц доказана.

Стоит заметить, что соотношения (А.6), (А.5) между константами связи $N, F, P, L$ не единственно возможные. Выбирая другие решения соотношения звезда-треугольник, мы получим дополнительные тождества, которым удовлетворяют трансфер-матрицы двумерной модели Изинга с разными граничными условиями.

ПРИЛОЖЕНИЕ Б

Доказательство связи гамильтонианов одномерной цепочки Изинга с трансфер-матрицами двумерных моделей Изинга

Докажем приведенный результат только для граничных условий $(++)$. Доказательство для остальных случаев абсолютно аналогично.

Пусть трансфер-матрица дается диаграммой, представленной на рис. 9.

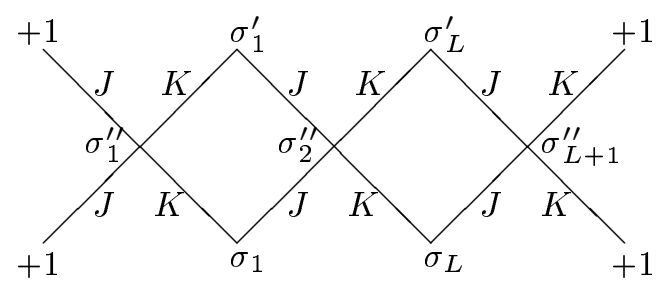

Рис. 9 
Очевидно, что $T(u)=V(u) W(u)$, где

$$
\begin{aligned}
V_{\Phi, \Phi^{\prime \prime}} & =\exp \left[\sum_{j=1}^{L-1}\left(K \sigma_{j} \sigma_{j}^{\prime \prime}+J \sigma_{j} \sigma_{j+1}^{\prime \prime}\right)+J \sigma_{1}^{\prime \prime}+K \sigma_{L}^{\prime \prime}\right], \\
W_{\Phi^{\prime \prime}, \Phi^{\prime}} & =\exp \left[\sum_{j=1}^{L-1}\left(K \sigma_{j}^{\prime} \sigma_{j}^{\prime \prime}+J \sigma_{j}^{\prime} \sigma_{j+1}^{\prime \prime}\right)+J \sigma_{1}^{\prime \prime}+K \sigma_{L}^{\prime \prime}\right]
\end{aligned}
$$

При $u=0$ имеем $K=0, J=\infty$. Найдем $d K /\left.d u\right|_{u=0}$ и $d J /\left.d u\right|_{u=0}$. Дифференцируя равенство $\operatorname{sh}(2 K)=\operatorname{tg} u$, получим

$$
2 \operatorname{ch}(2 K) \frac{d K}{d u}=\frac{1}{\cos ^{2} u}
$$

Полагая $u=0$, имеем отсюда

$$
\frac{d K}{d u}(0)=\frac{1}{2}
$$

Аналогично из равенства $\operatorname{sh}(2 J)=\operatorname{ctg} u$ получаем

$$
2 \operatorname{ch}(2 J) \frac{d J}{d u}=-\frac{1}{\sin ^{2} u} .
$$

При $u=0$ обе части равенства равны бесконечности. Поэтому, считая $J$ большим числом, имеем

$$
\frac{d J}{d u}(u \rightarrow 0)=-\frac{1}{2} \operatorname{ch}(2 J) .
$$

Поскольку $K=0$, то

$$
\begin{aligned}
V_{\Phi, \Phi^{\prime \prime}} & =\exp \left[\sum_{j=1}^{L-1} J \sigma_{j} \sigma_{j+1}^{\prime \prime}+J \sigma_{1}^{\prime \prime}\right]=\exp \left(J \sigma_{1}^{\prime \prime}\right) \prod_{j=1}^{L-1} \exp \left(J \sigma_{j} \sigma_{j+1}^{\prime \prime}\right), \\
W_{\Phi^{\prime \prime}, \Phi^{\prime}} & =\exp \left[\sum_{j=1}^{L-1} J \sigma_{j}^{\prime} \sigma_{j+1}^{\prime \prime}+J \sigma_{1}^{\prime \prime}\right]=\exp \left(J \sigma_{1}^{\prime \prime}\right) \prod_{j=1}^{L-1} \exp \left(J \sigma_{j}^{\prime} \sigma_{j+1}^{\prime \prime}\right) .
\end{aligned}
$$

Следовательно, трансфер-матрица есть

$$
\begin{aligned}
T_{\Phi, \Phi^{\prime}}(0) & =\sum_{\left\{\sigma^{\prime \prime}\right\}} \exp \left(2 J \sigma_{1}^{\prime \prime}\right) \prod_{j=1}^{L-1} \exp \left[J \sigma_{j+1}^{\prime \prime}\left(\sigma_{j}+\sigma_{j}^{\prime}\right)\right]= \\
& =2^{L} \operatorname{ch}(2 J) \prod_{j=1}^{L-1} \operatorname{ch}\left[J\left(\sigma_{j}+\sigma_{j}^{\prime}\right)\right]
\end{aligned}
$$

Найдем $T_{\Phi, \Phi^{\prime}}^{-1}(0)$. Поскольку $T_{\Phi^{\prime \prime}, \Phi^{\prime}}(0)$ есть произведение сомножителей вида $\operatorname{ch}\left(\sigma_{j}^{\prime \prime}+\right.$ $\left.\sigma_{j}^{\prime}\right)$, разумно искать обратную ей матрицу в виде произведения

$$
T_{\Phi, \Phi^{\prime \prime}}^{-1}(0)=\frac{1}{2^{L} \operatorname{ch}(2 J)} \prod_{j=1}^{L-1} A\left(\sigma_{j}^{\prime \prime}, \sigma_{j}\right)
$$


МИНИМАЛЬНАЯ РЕШЕТОЧНАЯ МОДЕЛЬ $L M(3,4)$ И ДВУМЕРНАЯ МОДЕЛЬ ИЗИНГА 81

Имеем

$$
\sum_{\left\{\sigma^{\prime \prime}\right\}} \operatorname{ch}\left[J\left(\sigma^{\prime \prime}+\sigma^{\prime}\right)\right] A\left(\sigma^{\prime \prime}, \sigma\right)=\delta\left(\sigma^{\prime}, \sigma\right),
$$

где $\delta\left(\sigma^{\prime}, \sigma\right)$ - символ Кронекера.

Производя суммирование по $\sigma^{\prime \prime}$, получим уравнение

$$
\operatorname{ch}\left[J\left(\sigma^{\prime}+1\right)\right] A(1, \sigma)+\operatorname{ch}\left[J\left(\sigma^{\prime}-1\right)\right] A(-1, \sigma)=\delta\left(\sigma^{\prime}, \sigma\right),
$$

откуда видно, что

$$
A\left(\sigma^{\prime \prime}, \sigma\right)=\frac{\operatorname{ch}\left[J\left(\sigma+\sigma^{\prime \prime}\right)\right]}{\operatorname{sh}^{2}(2 J)} \sigma \sigma^{\prime \prime}
$$

Поэтому

$$
T_{\Phi, \Phi^{\prime \prime}}^{-1}(0)=\frac{1}{2^{L} \operatorname{ch}(2 J)} \prod_{j=1}^{L-1} \frac{\operatorname{ch}\left[J\left(\sigma_{j}+\sigma_{j}^{\prime \prime}\right)\right]}{\operatorname{sh}^{2}(2 J)} \sigma_{j} \sigma_{j}^{\prime \prime} .
$$

Продиффференцируем $T(u)$ по $u$,

$$
\dot{T}(u)=\left(\frac{d V}{d K} \frac{d K}{d u}+\frac{d V}{d J} \frac{d J}{d u}\right) W+V\left(\frac{d W}{d K} \frac{d K}{d u}+\frac{d W}{d J} \frac{d J}{d u}\right) .
$$

Дифференцируя $V$ и $W$ по $J$ и полагая $u$ равным нулю, получим

$$
\begin{aligned}
\left(\frac{d V}{d J}\right)_{\Phi, \Phi^{\prime \prime}} & =\left(\sum_{k=1}^{L-1} \sigma_{k} \sigma_{k+1}^{\prime \prime}+\sigma_{1}^{\prime \prime}\right) \exp \left(J \sigma_{1}^{\prime \prime}\right) \prod_{j=1}^{L-1} \exp \left(J \sigma_{j} \sigma_{j+1}^{\prime \prime}\right), \\
\left(\frac{d W}{d J}\right)_{\Phi^{\prime \prime}, \Phi^{\prime}} & =\left(\sum_{k=1}^{L-1} \sigma_{k}^{\prime} \sigma_{k+1}^{\prime \prime}+\sigma_{1}^{\prime \prime}\right) \exp \left(J \sigma_{1}^{\prime \prime}\right) \prod_{j=1}^{L-1} \exp \left(J \sigma_{j}^{\prime} \sigma_{j+1}^{\prime \prime}\right) .
\end{aligned}
$$

Следовательно,

$$
\begin{aligned}
\left(\frac{d V}{d J} W\right)_{\Phi, \Phi^{\prime}}= & \sum_{\left\{\sigma^{\prime \prime}\right\}}\left(\sum_{k=1}^{L-1} \sigma_{k} \sigma_{k+1}^{\prime \prime}+\sigma_{1}^{\prime \prime}\right) \exp \left(2 J \sigma_{1}^{\prime \prime}\right) \prod_{j=1}^{L-1} \exp \left[J \sigma_{j+1}^{\prime \prime}\left(\sigma_{j}+\sigma_{j}^{\prime}\right)\right]= \\
= & 2^{L} \operatorname{ch}(2 J) \sum_{k=1}^{L-1} \sigma_{k} \operatorname{sh}\left[J\left(\sigma_{k}+\sigma_{k}^{\prime}\right)\right] \prod_{j=1, j \neq k}^{L-1} \operatorname{ch}\left[J\left(\sigma_{j}+\sigma_{j}^{\prime}\right)\right]+ \\
& +2^{L} \operatorname{sh}(2 J) \prod_{j=1}^{L-1} \operatorname{ch}\left[J\left(\sigma_{j}+\sigma_{j}^{\prime}\right)\right] .
\end{aligned}
$$

Аналогично получим

$$
\begin{aligned}
\left(V \frac{d W}{d J}\right)_{\Phi, \Phi^{\prime}}= & \sum_{\left\{\sigma^{\prime \prime}\right\}}\left(\sum_{k=1}^{L-1} \sigma_{k}^{\prime} \sigma_{k+1}^{\prime \prime}+\sigma_{1}^{\prime \prime}\right) \exp \left(2 J \sigma_{1}^{\prime \prime}\right) \prod_{j=1}^{L-1} \exp \left[J \sigma_{j+1}^{\prime \prime}\left(\sigma_{j}+\sigma_{j}^{\prime}\right)\right]= \\
= & 2^{L} \operatorname{ch}(2 J) \sum_{k=1}^{L-1} \sigma_{k}^{\prime} \operatorname{sh}\left[J\left(\sigma_{k}+\sigma_{k}^{\prime}\right)\right] \prod_{j=1, j \neq k}^{L-1} \operatorname{ch}\left[J\left(\sigma_{j}+\sigma_{j}^{\prime}\right)\right]+ \\
& +2^{L} \operatorname{sh}(2 J) \prod_{j=1}^{L-1} \operatorname{ch}\left[J\left(\sigma_{j}+\sigma_{j}^{\prime}\right)\right] .
\end{aligned}
$$


Сложим два последних равенства и умножим результат на $d J / d u$ :

$$
\begin{gathered}
\left(V \frac{d W}{d J}+\frac{d V}{d J} W\right)_{\Phi^{\prime \prime}, \Phi^{\prime}} \frac{d J}{d u}=2^{L} \operatorname{ch}(2 J) \sum_{k=1}^{L-1}\left(\sigma_{k}^{\prime \prime}+\sigma_{k}^{\prime}\right) \operatorname{sh}\left[J\left(\sigma_{k}^{\prime \prime}+\sigma_{k}^{\prime}\right)\right] \times \\
\times \prod_{j=1, j \neq k}^{L-1} \operatorname{ch}\left[J\left(\sigma_{j}^{\prime \prime}+\sigma_{j}^{\prime}\right)\right]+2^{L+1} \operatorname{sh}(2 J) \prod_{j=1}^{L-1} \operatorname{ch}\left[J\left(\sigma_{j}^{\prime \prime}+\sigma_{j}^{\prime}\right)\right] .
\end{gathered}
$$

Учитывая последние формулы, имеем

$$
\begin{aligned}
\sum_{\Phi^{\prime \prime}} T_{\Phi, \Phi^{\prime \prime}}^{-1} & \left(V \frac{d W}{d J}+\frac{d V}{d J} W\right)_{\Phi^{\prime \prime}, \Phi^{\prime}}\left(\frac{d J}{d u}\right)=-\operatorname{sh}(2 J)- \\
& -\frac{1}{2} \operatorname{ch}(2 J) \sum_{\sigma^{\prime \prime}} \sum_{k=1}^{L-1}\left(\sigma_{k}^{\prime \prime}+\sigma_{k}^{\prime}\right) \operatorname{sh}\left[J\left(\sigma_{k}^{\prime \prime}+\sigma_{k}^{\prime}\right)\right] \sigma_{k} \sigma_{k}^{\prime \prime} \frac{\operatorname{ch}\left[J\left(\sigma_{k}+\sigma_{k}^{\prime \prime}\right)\right]}{\operatorname{sh}^{2}(2 J)}= \\
= & -\frac{1}{2} \frac{\operatorname{ch}(2 J)}{\operatorname{sh}^{2}(2 J)} \sum_{k=1}^{L-1}\left[\left(\sigma_{k}^{\prime}+1\right) \sigma_{k} \operatorname{sh}\left[J\left(\sigma_{k}^{\prime}+1\right)\right] \operatorname{ch}\left[J\left(\sigma_{k}+1\right)\right]-\right. \\
& \left.-\left(\sigma_{k}^{\prime}-1\right) \sigma_{k} \operatorname{sh}\left[J\left(\sigma_{k}^{\prime}-1\right)\right] \operatorname{ch}\left[J\left(\sigma_{k}-1\right)\right]\right]-\operatorname{sh}(2 J)
\end{aligned}
$$

Легко видеть, что полученное выражение не расходится при $J \rightarrow \infty$, только если $\sigma_{k}=-\sigma_{k}^{\prime}$ (сингулярный член $\operatorname{sh}(2 J)$ мы не учитываем). Необходимо рассмотреть два случая:

1) $\sigma_{k}=-\sigma_{k}^{\prime}=+1$

2) $\sigma_{k}=-\sigma_{k}^{\prime}=-1$.

В первом случае правая часть выражения (Б.9) сводится к

$$
\frac{\operatorname{ch}(2 J)}{\operatorname{sh}^{2}(2 J)} \sum_{k=1}^{L-1} \operatorname{sh}(2 J) \rightarrow \sum_{k=1}^{L-1} 1
$$

при $J \rightarrow \infty$. Во втором случае - к

$$
\frac{\operatorname{ch}(2 J)}{\operatorname{sh}^{2}(2 J)} \sum_{k=1}^{L-1} \operatorname{sh}(2 J) \rightarrow \sum_{k=1}^{L-1} 1 .
$$

В результате мы видим, что несингулярную часть выражения (Б.9) можно записать в виде $\sum_{k=1}^{L-1} \sigma_{k}^{x}$ (матричные элементы в обоих случаях совпадают). Здесь мы ввели обозначение $\sigma_{k}^{x}=1 \otimes \cdots \otimes \sigma^{x} \otimes \cdots \otimes 1$, где оператор $\sigma^{x}$ действует на $k$-й спин цепочки.

Аналогичные вычисления для

$$
\left(V \frac{d W}{d K}+\frac{d V}{d K} W\right)_{\Phi^{\prime \prime}, \Phi^{\prime}}\left(\frac{d K}{d u}\right)
$$


приводят к выражению

$$
\sum_{k=1}^{L-2} \sigma_{k}^{z} \sigma_{k+1}^{z}+\sigma_{1}^{z}+\sigma_{L-1}^{z} .
$$

В результате несингулярная часть логарифмической производной трансфер-матрицы действительно оказывается равной гамильтониану:

$$
H_{\mathrm{I} \text { sing }}^{++}(L)=H_{\mathrm{Ising}}^{0}(L-1)+\sigma_{1}^{z}+\sigma_{L-1}^{z}
$$

Таким образом, связь гамильтониана одномерной цепочки Изинга с трансфер-матрицей доказана.

Благодарности. Мы благодарны П. Мартину, Б. Фейгину и особенно Ф. Алькарацу и П. Пирсу за полезные обсуждения.

\section{Список литературы}

[1] R. E. Behrend, P. A. Pearce, D. L. O'Brien. Interaction-round-a-face models with fixed boundary conditions: The ABF fusion hierarchy. Preprint University of Melbourne, hep-th/9507118.

[2] D. L. O'Brien, P. A. Pearce, S. Ole Warnaar. Physica A. 1996. V. 228. P. 63.

[3] F. Alcaraz, M. Barber, M. Batchelor. Phys. Rev. Lett. 1987. V. 58. P. 771; F. Alcaraz, M. Barber, M. Batchelor, R. Baxter, G. Quispel. J. Phys. A. 1987. V. 20. P. 6397.

[4] C. Hamer. J. Phys. A. 1981. V. 14. P. 2981.

[5] F. Alcaraz, M. Baake, U. Grimm, V. Rittenberg. J. Phys. A. 1989. V. 22. P. L5.

[6] V. Pasqier, H. Saleur. Nucl. Phys. B. 1990. V. 330. P. 523.

[7] A. Belavin, Yu. Stroganov. Minimal models of integrable lattice theory and truncated functional equations. hep-th/9908050.

[8] E. K. Sklyanin. J. Phys. A. 1988. V. 21. P. 2375.

[9] P.P. Kulish, E. K. Sklyanin. J. Phys. A. 1991. V. 24. P. L435.

[10] F. C. Alcaraz, A.A. Belavin, R.A. Usmanov. Correspondence between the $X X Z$ model in roots of unity and the one-dimensional quantum Ising chain with different boundary conditions. hep-th/0007151.

[11] E. Lusztig. Contemp. Math. 1989. V. 82. P. 59.

[12] P. P. Martin. Potts Models and Related Problems in Statistical Mechanics. Singapore: World Scientific, 1991. 